\title{
Thermal scaling laws of the optical Bragg acceleration structure
}

\author{
Vadim Karagodsky, Amit Mizrahi, and Levi Schächter* \\ Department of Electrical Engineering, Technion-Israel Institute of Technology, Haifa 32000, Israel
}

(Received 12 February 2006; published 23 May 2006)

\begin{abstract}
The temperature distribution and heat flow in the planar optical Bragg acceleration structure, fed by a train of high-power laser pulses, are analyzed. Dynamic analysis of a high-repetition rate train of pulses indicates that the stationary solution is an excellent approximation for the regime of interest. Analytic expressions for the temperature and heat distributions across the acceleration structure are developed. Assuming an accelerating gradient of $1 \mathrm{GV} / \mathrm{m}$ and a loss factor similar to that existing in communication optical fibers $1 \mathrm{~dB} / \mathrm{km}\left(\tan \delta \sim 10^{-11}\right)$, the temperature increase is less than $1 \mathrm{~K}$ and the heat flow is of the order of $1 \mathrm{~W} / \mathrm{cm}^{2}$, which is 3 orders of magnitude lower than the known technological limit for heat dissipation. Obviously, using materials with a significantly higher loss tangent may lead to unacceptable temperatures and temperature gradients as well as confinement difficulties and phase mismatch.
\end{abstract}

DOI: 10.1103/PhysRevSTAB.9.051301

PACS numbers: 29.17.+w, 81.70.Pg, 81.40.Gh, 41.75.Lx

\section{INTRODUCTION}

Motivated by the availability of solid-state lasers with increasing wall plug to light efficiencies, optical acceleration of charged particles is a subject of recent interest. Acceleration is facilitated by laser light rather than by microwave radiation, and accordingly, the acceleration structure must be made of dielectric materials as these have lower loss and are less susceptible to breakdown compared to their metallic counterparts. An example of an open optical structure is the LEAP [1] crossed laser beam experiment where the interaction between the crossed laser beams and the particles is limited by slits to satisfy the Lawson-Woodward theorem [2,3]. Another example is the traveling wave acceleration structure, where a laser pulse is guided in a dielectric structure with a vacuum tunnel bored in its center. This concept can be implemented by a two-dimensional photonic band-gap structure [4], and recently it was suggested [5] to use Bragg reflection waveguides [6-8], designed specifically for the speed-of-light mode.

In Ref. [5], it was demonstrated that optical Bragg acceleration structures, either planar or cylindrical, having typical transverse dimensions of a few microns, exhibit high performance as acceleration structures and, therefore, seem to be promising candidates for future optical accelerators. In this study, we focus on the planar optical Bragg acceleration structure illustrated in Fig. 1. The laser light is guided in a vacuum core of width $2 D_{\text {int }}$, so that the wave propagates along the $z$ axis, and no variations are assumed along the $y$ axis $(\partial / \partial y \equiv 0)$. The core is surrounded by dielectric layers with alternating permittivity, having a width equal to the transverse quarter-wavelength $\left[\lambda /\left(4 \sqrt{\varepsilon_{r}-1}\right)\right]$ with the exception of the innermost layer. This first layer is a matching layer whose width is deter-

*Electronic address: levi@ee.technion.ac.il mined so that the structure supports the speed-of-light TM mode required for the acceleration process [5].

Transition from operation at radiation wavelengths of a few centimeters to a few microns requires examining a wide spectrum of phenomena which are insignificant in the former regime. For example, structures that operate at a wavelength of a few centimeters are machined today with an accuracy of microns. In the future, it will not be possible to maintain a difference of 4-5 orders of magnitude between the operating wavelength and the achievable tolerance, since this would entail engineering of a surface at the atomic level. As a result, the size of irregularities may be of the same order of magnitude as the microbunches, and they may generate wake fields $[9,10]$ that, in turn, may alter the dynamics of electrons. Fortunately, the electromagnetic properties of materials at wavelengths that are significantly smaller than $0.1 \mu \mathrm{m}$ do not differ dramatically from these of the vacuum. Consequently, a reduction on the sensitivity to manufacturing tolerances may be expected.

Another aspect that may have a critical impact on the performance of a dielectric acceleration structure and will be investigated here is the heat dissipation and the temperature increase associated with electromagnetic power loss. As a central component of a future optical accelerator, the acceleration structure ought to withstand the manifes-

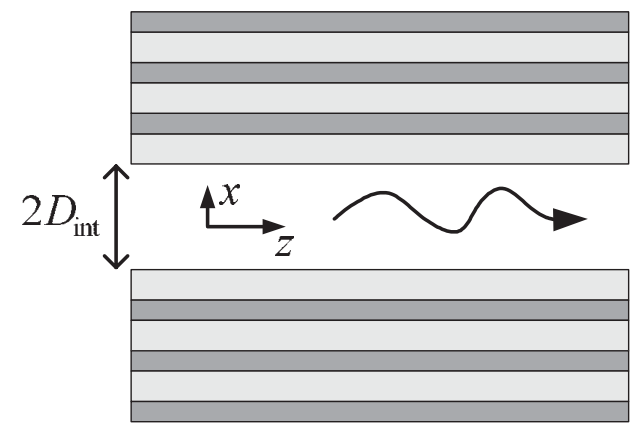

FIG. 1. Planar optical Bragg acceleration structure. 
tations of the most important constraint imposed by the machine specifications, namely, the luminosity. Being a measure of the number of colliding particles per second at the interaction point, the luminosity sets the lower limit to the energy level the particles are exposed to. Together with the constraint of single mode operation, they determine the minimal electromagnetic energy density in the acceleration tunnel and its close vicinity. While, obviously, the average number of particles per second is at least as in a machine designed to operate at microwave wavelengths, in an optical acceleration structure, the volume where most of the electromagnetic energy is confined is reduced by several orders of magnitude. Consequently, the potential damage due to heat flow or temperature increase may become a significant obstacle, and it is our goal in this study to determine the main scaling laws of these processes.

Before examining the heat dissipation in an acceleration structure, it is instructive to briefly review previous work on related topics. Attention to the thermal effects induced by the overheating of laser structures grew along with the progress made in high-power laser systems more than 20 years ago. Thermal considerations gradually became a limiting factor on the laser performance. Studies by Eggleston et al. [11] and Kane et al. [12] analyzed phenomena such as thermally induced stress, thermo-optical modification of the refractive index, birefringence, and thermal "lensing" in a slab geometry laser. The thermal stress and the refractive index modification [13,14], along with the birefringence $[15,16]$ and the thermal lensing $[17,18]$, were further investigated. Evidently, the damage caused by the thermal effects can be controlled by proper cooling. A number of practical cooling setups for laser systems were experimentally tested [19,20]. Diamond films proved to be particularly useful in cooling schemes, being an excellent heat conducting material $[21,22]$.

Two traditional theoretical models describe the cooling process. In the first case, the so-called Newton's law of heat transfer, the amount of heat extracted from a cooled surface is proportional to the temperature difference between the surface and the coolant. The proportionality factor $h$ is the heat transfer coefficient, which is a measure of the cooling efficiency. Xie et al. [23-25] studied the factors that affect the heat transfer coefficient and its relation to the thermal effects inside the laser structure. The second theoretical model describing the cooling process is the heat sink, according to which the temperature at the outer surface is identical to the temperature of the adjacent heat sink. This is, in fact, the first model in the limit $h \rightarrow \infty$.

Analysis of thermal effects requires establishing the temperature distribution inside the structure. Koechner [26] was among the first to derive single pulse and multipulse solutions for an infinitely long and pumped uniformly in the longitudinal direction laser rod. A more realistic analysis, considering single and multipulse tem- perature distributions in a finite rod with an arbitrary distribution in the longitudinal direction of the pumping energy, is given in Refs. $[27,28]$. The steady-state solution for a finite laser slab was developed [29], and the steadystate problem of a laser rod subject to a cylindrically symmetric and longitudinally homogeneous pumping was solved [16]. The steady-state heat distribution caused by longitudinally inhomogeneous pumping was recently studied as well [30].

All studies mentioned above consider single-layered structures. Heat conduction in multilayered structures has also been investigated. For example, the thermal profile of a multilayered structure heated by a scanning laser or by an electron beam [31-34] has been determined, both being common processes in fields such as magneto-optical media, electron beam lithography, and ion implantation. A similar problem was solved in order to describe the photothermal deflection of a laser beam passing above heated multilayered media [35]. In recent years, there has been an increasing interest in the heat transfer in multilayered structures [36-39]. In Ref. [36], a solution of the heat conduction equation for a two-layered cylindrical structure heated by a short laser pulse was introduced. The transient [37] and the steady-state [38,39] 3-dimensional heat conduction in multilayered structures was studied. Further extensive analysis on the transient heat transfer in multilayered structures was also performed recently [40-43].

In the present study, we aim to determine the scaling laws regarding heat flow and temperature increase in the planar optical Bragg acceleration structure. We focus on the temperature developed due to the propagating laser, rather than including the wakefield as a source of heat dissipation and consequently obtaining a temperature rise. The part of the wakefield that propagates at the fundamental speed-of-light mode is therefore tacitly included in the estimation given here, whereas the high frequency content of the wake is not expected to significantly change the general picture. In the next section, we examine the thermal energy flow in the structure illustrated in Fig. 1 when a train of laser pulses in injected into it. Each pulse is assumed to have an electromagnetic power profile which is based on an analytic estimate. Assuming similar thermal properties to the various layers, it is demonstrated that, for the set of parameters of interest, the deviation of the peak temperature or heat flow from the time-averaged value is extremely small. Analytic expressions for the average temperature and heat flow are developed. These are the important quantities that provide an excellent description of the thermal process since the latter occurs on a much longer time scale comparing to the electromagnetic processes.

In Sec. III, we establish the time-averaged temperature and heat flow in the Bragg structure and develop corresponding analytic expressions. In Sec. IV, we discuss two possible configurations using the tools developed in 
Sec. III. In the last section, we briefly examine the thermal stress developed in the dielectric layers of the Bragg acceleration structure.

\section{APPROXIMATE DYNAMIC ANALYSIS}

Consider the planar optical Bragg acceleration structure shown in Fig. 1. The structure may support an accelerating gradient of the order of $E_{A} \simeq 1 \mathrm{GV} / \mathrm{m}$ at a typical radiation wavelength of $\lambda_{0} \simeq 1 \mu \mathrm{m}$. We assume that a train of laser pulses is injected into the acceleration structure, and it is the goal of this study to establish the temperature dynamics within the structure. For simplicity, it is assumed in this section that the dielectric layers have identical thermal characteristics (single layer), and the dissipated power across the device is approximated by its general behavior as given in Ref. [5]. Consequently, we may develop an analytic result, which is an approximation of the exact solution. In the following section, the different thermal characteristics of the layers and the exact behavior of the electromagnetic field are accounted for.

The power propagates at the speed-of-light mode, having a transverse profile $X_{0}(x)$, which is determined by the detailed geometric and electrical characteristics of the structure as well as the vacuum wavelength. For the planar optical Bragg acceleration structure, the transverse profile may be approximated by [5]

$$
X_{0}(x) \simeq \exp \left[-2 \frac{x-D_{\text {int }}}{x_{c}}\right]
$$

where $\quad x_{c}=\left(\lambda_{0} / 4\right)\left[\left(\varepsilon_{1}-1\right)^{-1 / 2}+\left(\varepsilon_{2}-1\right)^{-1 / 2}\right] \times$ $\left|\ln \left[\varepsilon_{1}\left(\varepsilon_{1}-1\right)^{-1 / 2} / \varepsilon_{2}\left(\varepsilon_{2}-1\right)^{-1 / 2}\right]\right|^{-1} ; \varepsilon_{1}$ is the permittivity of the layer adjacent to the core, and the remainder of the layers are alternating with materials $\varepsilon_{2}$ and $\varepsilon_{1}$. Each pulse has a time duration $T_{\mathrm{p}}$ which is of the order of picoseconds, and they are separated by intervals of $T_{\text {rr }}$ that, in turn, may vary from microseconds to nanoseconds. The pulses travel at a group velocity $V_{\mathrm{gr}}$, and have a temporal profile of $T_{0}(t)$, so that for the simple case of a rectangular pulse, the temporal profile of the power density may be represented as a Fourier series $T_{0}(t)=$ $\sum_{\nu} f_{\nu} \exp \left(j 2 \pi \nu t / T_{\text {rr }}\right), \quad$ where $\quad f_{\nu}=\left(T_{\mathrm{p}} / T_{\text {rr }}\right) \times$ $\operatorname{sinc}\left(\pi \nu T_{\mathrm{p}} / T_{\text {rr }}\right)$ and $\operatorname{sinc}(\xi) \equiv \sin (\xi) / \xi$. The temperature developed inside the structure is due to the dissipated electromagnetic power given by

$$
P_{\text {loss }}=\frac{1}{2} \omega \varepsilon_{0} \varepsilon_{r} \tan \delta\left(\left|E_{x}^{2}\right|+\left|E_{z}^{2}\right|\right)
$$

where $\tan \delta$ is the material's loss tangent. Consequently, the dissipated power may be written in the form $P_{\text {loss }}=$ $P_{0} X_{0}(x) T_{0}\left(t-z / V_{\mathrm{gr}}\right)$. Based on Ref. [5], the field in the vacuum tunnel, having a time dependence of $e^{j \omega t}$, is of the form $E_{z}=E_{\mathrm{A}} \exp \left(-j \frac{\omega}{c} z\right)$, and accordingly, the peak value of the dissipated power may be approximated by

$$
P_{0} \simeq \frac{1}{2} \omega \varepsilon_{0} \varepsilon_{1} \tan \delta E_{\mathrm{A}}^{2}\left[1+\left(\frac{1}{\varepsilon_{1}} \frac{\omega}{c} D_{\mathrm{int}}\right)^{2}\right]
$$

note that the expressions in Eqs. (2) and (3) tacitly assume time average over one period of the radiation field.

All the characteristic time parameters of the electromagnetic problem are orders of magnitude shorter than that corresponding to the diffusion process. The latter controls the temperature variation $\Delta T$ throughout the system described by

$$
\left(\frac{\partial}{\partial t}-\frac{1}{D} \nabla^{2}\right) \Delta T=\frac{1}{D \sigma_{\mathrm{T}}} P_{\text {loss }},
$$

where $D$ is the thermal diffusion coefficient, and $\sigma_{\mathrm{T}}$ is the thermal conductivity. It is assumed that at the boundary of the outermost layer $x=D_{\text {ext }}$ the structure is in thermodynamic equilibrium with a "heat sink" which maintains a constant temperature at that boundary, i.e., $\Delta T(x=$ $\left.D_{\text {ext }}\right)=0$. In addition, there is no heat flow from the dielectric to the vacuum core, and thus we ignore radiative heat transfer and assume $[\partial \Delta T / \partial x]\left(x=D_{\text {int }}\right)=0$. Subject to the above conditions, the diffusion equation may be solved to obtain

$$
\begin{aligned}
\Delta T(x, z ; t)= & \frac{P_{0}}{\sigma_{\mathrm{T}}} \frac{T_{\mathrm{p}}}{T_{\mathrm{rr}}} \sum_{\nu=-\infty}^{\infty} \operatorname{sinc}\left(\pi \nu \frac{T_{\mathrm{p}}}{T_{\mathrm{rr}}}\right) \exp \left(j 2 \pi \nu \frac{t-z / V_{\mathrm{gr}}}{T_{\mathrm{rr}}}\right) \sum_{m=0}^{\infty} \cos \left[\frac{\pi(m+1 / 2)}{D_{\mathrm{ext}}-D_{\mathrm{int}}}\left(x-D_{\mathrm{int}}\right)\right] \frac{2}{D_{\mathrm{ext}}-D_{\mathrm{int}}} \\
& \times\left[j \frac{2 \pi \nu}{T_{\mathrm{rr}}} D+\left(\frac{2 \pi \nu}{T_{\mathrm{rr}}} \frac{1}{V_{\mathrm{gr}}}\right)^{2}+\frac{\pi^{2}(m+1 / 2)^{2}}{\left(D_{\mathrm{ext}}-D_{\mathrm{int}}\right)^{2}}\right]^{-1} \int_{D_{\mathrm{int}}}^{D_{\mathrm{ext}}} \mathrm{d} x^{\prime} X_{0}\left(x^{\prime}\right) \cos \left[\frac{\pi(m+1 / 2)}{D_{\text {ext }}-D_{\text {int }}}\left(x^{\prime}-D_{\text {int }}\right)\right] .
\end{aligned}
$$

The transverse heat flow may be derived from the temperature by $Q=-\sigma_{\mathrm{T}} \partial \Delta T / \partial x$. Two quantities of interest are readily evaluated: the average change in temperature $\left(\Delta T_{\mathrm{AV}}\right)$ over the repetition rate period of the pulse $\left(T_{\mathrm{rr}}\right)$ at the vacuumdielectric interface $\left(x=D_{\text {int }}\right)$, and second, the average heat flow $\left(Q_{\mathrm{AV}}\right)$ at the external layer $\left(x=D_{\text {ext }}\right)$. These two quantities were evaluated to read 


$$
\begin{aligned}
\Delta T_{\mathrm{AV}} & =\left[\frac{P_{0}}{\sigma_{\mathrm{T}}} \frac{x_{c}}{2}\left(D_{\mathrm{ext}}-D_{\mathrm{int}}\right)\right] \frac{T_{\mathrm{p}}}{T_{\mathrm{rr}}}\left[1-\frac{x_{c}}{2\left(D_{\mathrm{ext}}-D_{\mathrm{int}}\right)}\right] \sim \frac{P_{0}}{\sigma_{\mathrm{T}}} \frac{x_{c}}{2}\left(D_{\mathrm{ext}}-D_{\mathrm{int}}\right) \frac{T_{\mathrm{p}}}{T_{\mathrm{rr}}} \\
Q_{\mathrm{AV}} & =\left(P_{0} \frac{x_{c}}{2}\right) \frac{T_{\mathrm{p}}}{T_{\mathrm{rr}}},
\end{aligned}
$$

where the right-hand side of the top equation was evaluated assuming that $D_{\text {ext }}-D_{\text {int }} \gg x_{c} / 2$, a condition that may be dictated in part by the requirement for mechanical strength of the structure. Assuming $\left(D_{\text {ext }}-D_{\text {int }}\right) \gg x_{c} / 2$, the thermal transconductance has a very simple form, $Q_{\mathrm{AV}} / \Delta T_{\mathrm{AV}} \simeq \sigma_{\mathrm{T}} /\left(D_{\mathrm{ext}}-D_{\text {int }}\right)$, which is virtually independent of the electromagnetic characteristics of the structure $\left(x_{c}\right)$. In order to investigate the above results, we shall consider a silica-zirconia structure $\left(\varepsilon_{1}=2.1, \varepsilon_{2}=4\right)$ with a set of parameters given in Table I. Figure 2 illustrates the two quantities $Q_{\mathrm{AV}}$ and $T_{\mathrm{AV}}$ as a function of the core halfwidth $D_{\text {int }}$. The curves are computed assuming that the field on axis remains constant $\left(E_{A}=1 \mathrm{GV} / \mathrm{m}\right)$, so that the dissipated power $P_{0}$ of Eq. (6) changes with $D_{\text {int }}$ according to Eq. (3). Both quantities are linearly dependent on

TABLE I. Parameters used for the simulations shown in Figs. 2-4.

\begin{tabular}{lccc}
\hline \hline \multicolumn{2}{c}{ Structure parameters } & \multicolumn{2}{c}{ Pulse and thermal parameters } \\
\hline$\lambda_{0}$ & $1 \mu \mathrm{m}$ & $T_{\mathrm{p}}$ & $1 \mathrm{psec}$ \\
$\varepsilon_{1}, \varepsilon_{2}$ & $2.1,4.0$ & $T_{\mathrm{rr}}$ & $1 \mathrm{nsec}$ \\
$x_{c}$ & $2.68 \mu \mathrm{m}$ & $\sigma_{T}$ & $1.4 \mathrm{~W} / \mathrm{m} \mathrm{K}$ \\
$D_{\text {ext }}$ & $10 \mu \mathrm{m}$ & $D$ & $1.2 \times 10^{6} \mathrm{~s} / \mathrm{m}^{2}$ \\
\hline \hline
\end{tabular}

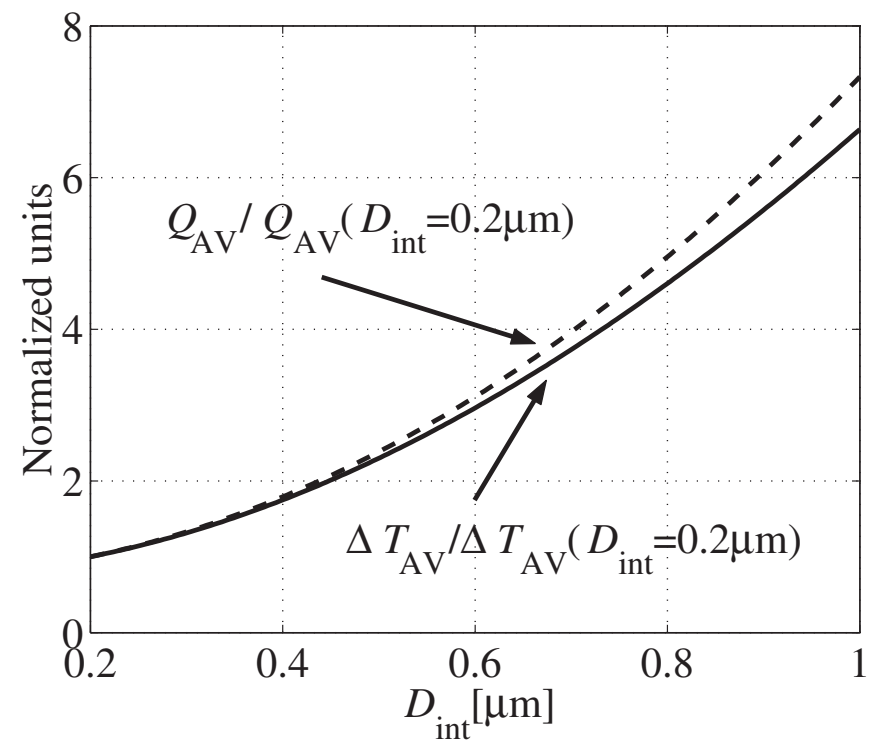

FIG. 2. The average change in temperature $\Delta T_{\mathrm{AV}}$ and the average heat flow $Q_{\mathrm{AV}}$ as a function of $D_{\text {int }}$, for a silica-zirconia structure with the parameters given in Table I. Both quantities are normalized by their value at $D_{\text {int }}=0.2 \mu \mathrm{m}, \Delta T_{\mathrm{AV}}\left(D_{\text {int }}=\right.$ $0.2 \mu \mathrm{m})=1.94 \times 10^{8} \tan \delta[\mathrm{K}]$ and $Q_{\mathrm{AV}}\left(D_{\text {int }}=0.2 \mu \mathrm{m}\right)=$ $3.21 \times 10^{9} \tan \delta\left[\mathrm{W} / \mathrm{cm}^{2}\right]$. the material loss tangent and their value at $D_{\text {int }}=$ $0.2 \mu \mathrm{m}$ is $\Delta T_{\mathrm{AV}}=1.94 \times 10^{8} \tan \delta[\mathrm{K}]$, and $Q_{\mathrm{AV}}=$ $3.2 \times 10^{9} \tan \delta\left[\mathrm{W} / \mathrm{cm}^{2}\right]$. Evidently, for the range of core half-widths shown in Fig. 2, if $\tan \delta<10^{-8}$, the temperature rise in the structure is less than $15 \mathrm{~K}$, and the average heat flow is about $250 \mathrm{~W} / \mathrm{cm}^{2}$.

While the average quantities $\left(\Delta T_{\mathrm{AV}}, Q_{\mathrm{AV}}\right)$ do not seem to pose a difficulty provided that $\tan \delta \leq 10^{-8}$, it still

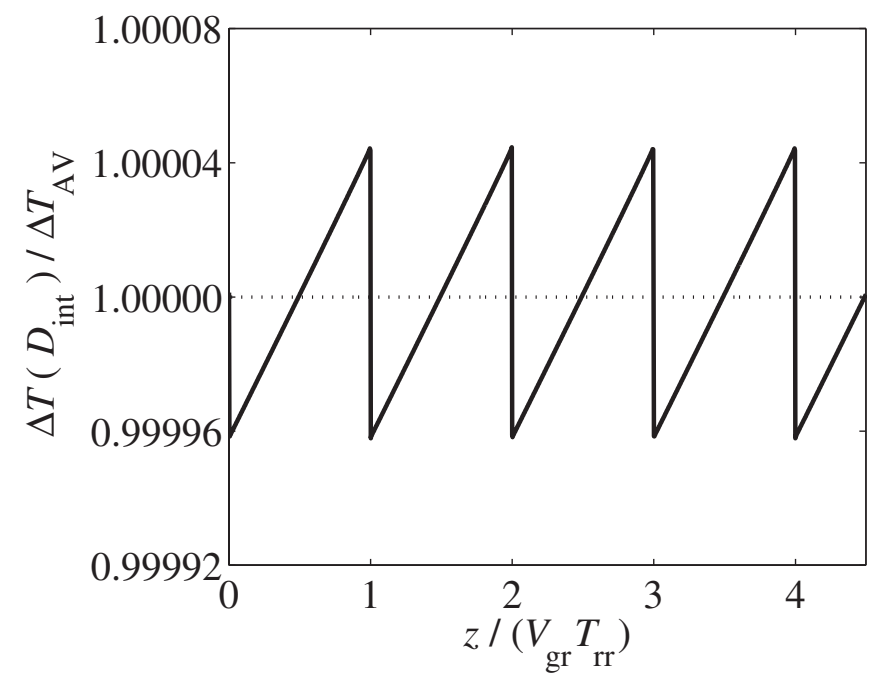

FIG. 3. A snapshot $(t=0)$ of the temperature variation at $x=$ $D_{\text {int }}$ normalized by its average value as a function of $z$. The core half-width is $D_{\text {int }}=0.6 \mu \mathrm{m}$, and the other parameters are given in Table I.

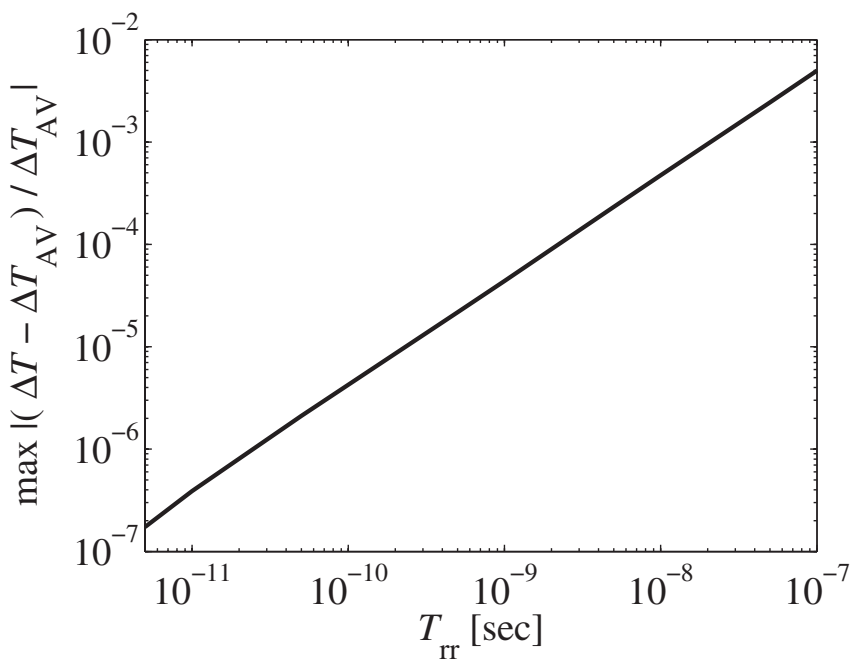

FIG. 4. The relative change in temperature $\max \mid(\Delta T-$ $\left.\Delta T_{\mathrm{AV}}\right) / \Delta T_{\mathrm{AV}}$ l as a function of the pulse separation $T_{\mathrm{rr}}$. The structure parameters excluding $T_{\mathrm{rr}}$ are given in Table I. 
remains to determine whether the peak temperature or heat flow do not impose any stringent constraints. For this purpose, the expression in Eq. (5) is plotted in Fig. 3, showing a snapshot of the temperature at the core boundary as a function of the longitudinal coordinate, for a structure having the parameters listed in Table I and $D_{\text {int }}=0.6 \mu \mathrm{m}$. The decay of the temperature behind each pulse is linear, as the diffusion time for this structure is of the order of $10^{-4} \mathrm{~s}$, thus much larger than $T_{\text {rr }}$ and $T_{\mathrm{p}}$, and the maximal relative change in temperature, $\max \mid(\Delta T-$ $\left.\Delta T_{\mathrm{AV}}\right) / \Delta T_{\mathrm{AV}} \mid<10^{-4}$, is extremely small. This last quantity is depicted in Fig. 4 for different values of pulse periodicity $T_{\mathrm{rr}}$, while the remainder of the parameters are as given in Table I. For a higher repetition rate (shorter $T_{\text {rr }}$ ), the excitation is closer to a $\mathrm{cw}$ signal, and therefore the temperature fluctuations are smaller. Even for a repetition rate of $10 \mathrm{MHz}\left(T_{\mathrm{rr}}=10^{-7} \mathrm{~s}\right)$, the relative change in temperature is less than $1 \%$. Clearly, for most practical purposes the deviation from the average values is miniscule. Therefore, in what follows, only the dc component $(v=0)$ in Eq. (5) will be considered.

\section{STEADY-STATE SOLUTION}

In the previous section, it was established, based on a simplified model, that at the high-repetition rate regime, the resulting temperature is to a very good approximation the solution of the steady-state problem, in which a cw signal is injected into the waveguide. In this section, the steady-state solution $(\partial / \partial t \equiv 0)$ of the diffusion equation [Eq. (4)], which becomes Poisson's equation, is developed for the exact configuration. Evidently, the steady-state solution of the heat dissipation problem is independent of the longitudinal coordinate $(\partial / \partial z \equiv 0)$. Moreover, for developing a realistic solution, a convective cooling mechanism is taken into consideration.

\section{A. Exact steady-state solution}

At this point, we are in a good position to establish the impact of the detailed geometry which facilitates the electromagnetic field confinement (see Fig. 1) on the steadystate solution. In each dielectric layer, the longitudinal electric field is of the form [5]

$$
E_{z, n}=\left[A_{n} \exp \left(-j k_{n} x\right)+B_{n} \exp \left(j k_{n} x\right)\right] \exp \left(-j \frac{\omega}{c} z\right)
$$

and, assuming that the field is confined, the amplitudes may be derived by imposing the boundary conditions, beginning from the vacuum core, where the field profile is known; $k_{n}=(\omega / c) \sqrt{\varepsilon_{r, n}-1}$ is the transverse wave number in the $n$th layer. With that in mind, the explicit form of the average dissipated power density is

$$
\begin{aligned}
P_{\text {loss }, n}= & \frac{1}{2} \omega \varepsilon_{0} \varepsilon_{r, n} \tan \delta_{n}\left[\frac{\varepsilon_{r, n}}{\varepsilon_{r, n}-1}\left(\left|A_{n}\right|^{2}+\left|B_{n}\right|^{2}\right)\right. \\
& \left.+\frac{\varepsilon_{r, n}-2}{\varepsilon_{r, n}-1} 2 \Re\left(A_{n}^{*} B_{n} \exp \left(2 j k_{n} x\right)\right)\right] .
\end{aligned}
$$

In each layer, Poisson's equation with the time-averaged dissipated power density term $\frac{1}{D \sigma_{\mathrm{T}}} P_{\text {loss }} \frac{T_{\mathrm{p}}}{T_{\mathrm{rr}}}$ must be solved, so that the change in the temperature in the $n$th layer satisfies

$$
\frac{\partial^{2}}{\partial x^{2}} \Delta T_{\mathrm{SS}, n}=-\frac{1}{\sigma_{\mathrm{T}, n}} \frac{T_{\mathrm{p}}}{T_{\mathrm{rr}}} P_{\mathrm{loss}, n}(x) .
$$

At the transition from one layer to another, both the temperature change and the heat flow ought to be continuous. Special attention is required at the vacuum-dielectric interface and at the outermost layer. The former is left as in the dynamic analysis case, namely,

$$
\left.\frac{\partial \Delta T_{\mathrm{SS}, 1}}{\partial x}\right|_{x=D_{\mathrm{int}}}=0
$$

For the external layer, we shall generalize the idealized assumption of a heat sink $\left.\Delta T_{\mathrm{SS}, N}\right|_{x=D_{\mathrm{ext}}}=0$ to include the impact of a convective cooling process; therefore

$$
\left.\Delta T_{\mathrm{SS}, N}\right|_{x=D_{\mathrm{ext}}}=-\left.\frac{\sigma_{\mathrm{T}, N}}{h} \frac{\partial \Delta T_{\mathrm{SS}, N}}{\partial x}\right|_{x=D_{\mathrm{ext}}},
$$

$h$ being the convective heat transfer coefficient, and values of 3 to $20 \mathrm{~W} / \mathrm{cm}^{2} \mathrm{~K}$ are considered in what follows. Values of this order were demonstrated to be achievable in cooling schemes of microelectronic devices [44-47].

\section{B. Approximate steady-state analytic solution}

In addition to the exact solution presented above, it is instructive to develop an approximate analytic expression relying on Eq. (1) and solving Eq. (9) subject to the boundary conditions in Eqs. (10) and (11). This is similar to the one layer model discussed in Sec. II, but only the dc term $[\nu=0$ in Eq. (5)] is taken here, and, in addition, the presence of the various layers is accounted for effectively. For this purpose, we shall assume a uniform thermal resistance, which is the serial resistance of the two layers, namely, we may define the effective thermal conductivity $\left(\sigma_{\mathrm{T}, \mathrm{eff}}\right)$ as $\frac{\Delta_{1}+\Delta_{2}}{\sigma_{\mathrm{T}, \text { eff }}}=\frac{\Delta_{1}}{\sigma_{\mathrm{T}, 1}}+\frac{\Delta_{2}}{\sigma_{\mathrm{T}, 2}}$. The analytic expressions for the local change in temperature and heat flow read 


$$
\begin{aligned}
\Delta T_{\mathrm{SS}, \mathrm{app}}(x)= & \frac{P_{0}}{\sigma_{\mathrm{T}, \mathrm{eff}}} \frac{T_{\mathrm{p}}}{T_{\mathrm{rr}}} \frac{x_{c}}{2}\left\{\left(D_{\text {ext }}-x\right)-\frac{x_{c}}{2}\left[\exp \left(-2 \frac{x-D_{\text {int }}}{x_{c}}\right)-\exp \left(-2 \frac{D_{\text {ext }}-D_{\text {int }}}{x_{c}}\right)\right]\right. \\
& \left.+\frac{\sigma_{\mathrm{T}, \mathrm{eff}}}{h}\left[1-\exp \left(-2 \frac{D_{\text {ext }}-D_{\text {int }}}{x_{c}}\right)\right]\right\} \\
Q_{\mathrm{SS}, \text { app }}(x)= & P_{0} \frac{T_{\mathrm{p}}}{T_{\text {rr }}} \frac{x_{c}}{2}\left[1-\exp \left(-2 \frac{x-D_{\text {int }}}{x_{c}}\right)\right],
\end{aligned}
$$

and, consequently,

$$
\begin{aligned}
\Delta T_{\mathrm{SS}, \text { app }}\left(x=D_{\text {int }}\right) & =\frac{P_{0}}{\sigma_{\mathrm{T}, \mathrm{eff}}} \frac{T_{\mathrm{p}}}{T_{\mathrm{rr}}} \frac{x_{c}}{2}\left(D_{\mathrm{ext}}-D_{\mathrm{int}}\right)\left(1-\frac{x_{c} / 2-\sigma_{\mathrm{T}, \mathrm{eff}} / h}{D_{\mathrm{ext}}-D_{\text {int }}}\right) \\
Q_{\mathrm{SS}, \text { app }}\left(x=D_{\text {ext }}\right) & =P_{0} \frac{T_{\mathrm{p}}}{T_{\mathrm{rr}}} \frac{x_{c}}{2},
\end{aligned}
$$

which for $h \rightarrow \infty$ are identical to those of a uniform layer discussed above [Eq. (6)]. The term $\left(1-\frac{x_{c} / 2-\sigma_{\text {T,eff }} / h}{D_{\text {ext }}-D_{\text {int }}}\right)$ in Eq. (13) expresses the temperature rise above the value dictated by the heat sink idealization, caused by a suboptimal convective coolant.

The approximate solution may be further improved by using $\kappa P_{0}$ instead of $P_{0}$, where $\kappa$ is a form factor given by

$$
\kappa=\frac{\int_{D_{\text {int }}}^{D_{\text {ext }}} \mathrm{d} x P_{\text {exact }}(x)}{\int_{D_{\text {int }}}^{D_{\text {ext }}} \mathrm{d} x P_{0} \exp \left(-2 \frac{x-D_{\text {int }}}{x_{c}}\right)},
$$

$P_{\text {exact }}(x)$ being the exact dissipated power profile according to Eq. (8). By this, we require that the total dissipated power across the structure is identical in the approximate and the exact expressions, and since this is the source of the temperature rise, a better approximation is obtained.

\section{ANALYSIS OF TWO CONFIGURATIONS}

Silica-zirconia. - In this section, we examine the temperature distribution and heat flow as obtained by the two solution methods introduced in the previous section. Figure 5 illustrates the steady-state temperature variation across the silica-zirconia acceleration structure for a set of parameters listed in Table II. The exact solution (Sec. III A) is represented by square markers, and the approximate solution (Sec. III B) is represented by a dashed line-the two are virtually indistinguishable. For the simulation purposes of the exact solution, it was assumed that the loss tangent of each material is the one corresponding to a conventional optical fiber made of that material, and having an attenuation of $\alpha[\mathrm{dB} / \mathrm{km}] \sim 1$. Attenuation coefficients of this order and less were, in fact, measured in silica fibers $[48,49]$. For convenience, we illustrate also the exact dissipated electromagnetic power profile, as evaluated based on Ref. [5], and the exponential decay approximation as given in Eq. (1). Each quantity is normalized by its maximal approximate value; the relevant normalization constants are listed in Table II. The maximum values are given in terms of the loss tangent of the first material (silica) denoted by $\tan \delta_{1}$. The accuracy of the approximate solution of the temperature is better than $0.5 \%$ relative to the temperature maximal value, and the accuracy of the approximate heat-flux solution, shown in Fig. 6, is better than $2 \%$ relative to its maximal value.

Three values of the convective heat transfer coefficient $h$ were examined, showing the role of the cooling mechanism in reducing the maximal temperature (see Table II). For the unnormalized curves, the effect of a different $h$ is to add an offset to the entire curve according to Eq. (12). However, the heat-flux profile, which is depicted in Fig. 6, is independent of $h$, as revealed by the approximate analytic expression of Eq. (12). Moreover, the heat flow is independent of the thermal conductivities, since according to Eq. (9), in conjunction with $Q_{\mathrm{SS}}=-\sigma_{\mathrm{T}} \partial \Delta T_{\mathrm{AV}} / \partial x$, we may readily conclude that the heat flow satisfies the equation $\partial Q_{\mathrm{SS}} / \partial x=\left(T_{\mathrm{p}} / T_{\text {rr }}\right) P_{\text {loss }}(x)$, subject to the vacuum-

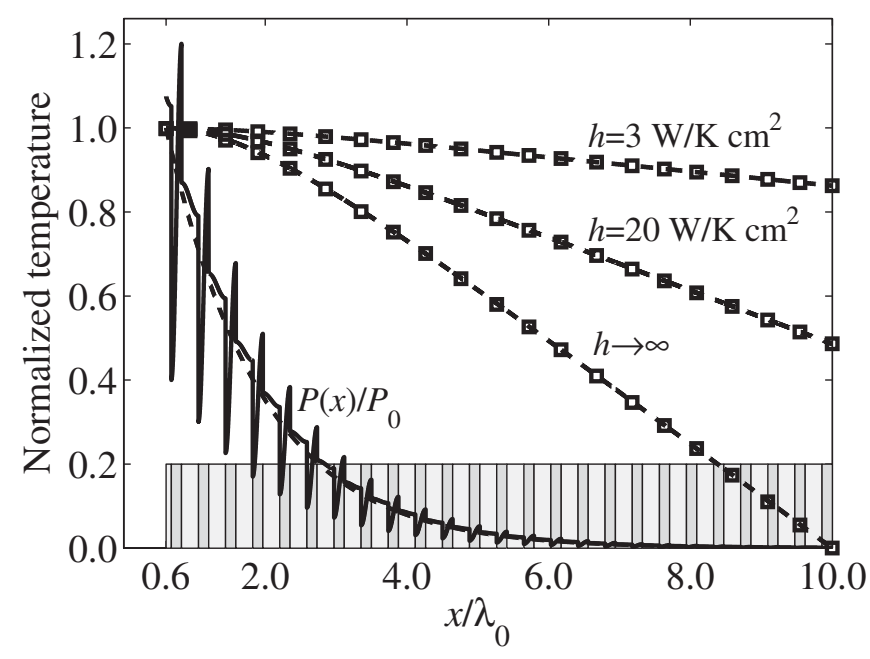

FIG. 5. Temperature profile for different values of $h$ in the silica-zirconia structure, the parameters of which are given in Table II. For each value of $h$, the dashed line is the approximate curve, and the exact solution is indicated by square markers. The dissipated power profiles, approximate (dashed line) and exact (solid line), are also plotted. All quantities are normalized by their maximal approximate value. 
TABLE II. Parameters used for the simulations presented in Figs. 5-8, and maximum values of the temperature and heat flux.

\begin{tabular}{|c|c|c|c|c|}
\hline & \multicolumn{2}{|c|}{ Silica-zirconia structure } & \multicolumn{2}{|c|}{ Silica-silicon structure } \\
\hline & $\mathrm{SiO}_{2}$ & $\mathrm{ZrO}_{2}$ & $\mathrm{SiO}_{2}$ & $\mathrm{Si}$ \\
\hline$\lambda_{0}$ & \multicolumn{4}{|c|}{$1 \mu \mathrm{m}$} \\
\hline$D_{\text {int }}$ & \multicolumn{4}{|c|}{$0.6 \mu \mathrm{m}$} \\
\hline$D_{\text {ext }}$ & \multicolumn{4}{|c|}{$\sim 10 \mu \mathrm{m}$} \\
\hline$T_{\mathrm{p}}\left(T_{\mathrm{rr}}\right)$ & \multicolumn{4}{|c|}{1 psec $(1$ nsec $)$} \\
\hline$\varepsilon_{r}$ & 2.1 & 4.0 & 2.1 & 11.6 \\
\hline$x_{c}$ & \multicolumn{2}{|c|}{$2.68 \mu \mathrm{m}$} & \multicolumn{2}{|c|}{$0.55 \mu \mathrm{m}$} \\
\hline$\kappa$ & \multicolumn{2}{|c|}{0.93} & \multicolumn{2}{|c|}{0.86} \\
\hline$\sigma_{\mathrm{T}}$ & $1.4 \mathrm{~W} / \mathrm{m} \mathrm{K}$ & $1.8 \mathrm{~W} / \mathrm{m} \mathrm{K}$ & $1.4 \mathrm{~W} / \mathrm{m} \mathrm{K}$ & $148 \mathrm{~W} / \mathrm{m} \mathrm{K}$ \\
\hline $\tan \delta @ 1 \mu \mathrm{m}$ & $2.5 \times 10^{-11}$ & $1.8 \times 10^{-11}$ & $2.5 \times 10^{-11}$ & $1.1 \times 10^{-11}$ \\
\hline$Q_{\max }$ & \multicolumn{2}{|c|}{$9.22 \times 10^{9} \tan \delta_{1}$} & \multicolumn{2}{|c|}{$1.74 \times 10^{9} \tan \delta_{1}$} \\
\hline$\Delta T_{\max }\left(h=3 \mathrm{~W} / \mathrm{K} \mathrm{cm}^{2}\right)$ & \multicolumn{2}{|c|}{$356 \times 10^{7} \tan \delta_{1}$} & \multicolumn{2}{|c|}{$66.4 \times 10^{7} \tan \delta_{1}$} \\
\hline$\Delta T_{\max }\left(h=20 \mathrm{~W} / \mathrm{K} \mathrm{cm}^{2}\right)$ & \multicolumn{2}{|c|}{$94.7 \times 10^{7} \tan \delta_{1}$} & \multicolumn{2}{|c|}{$17.2 \times 10^{7} \tan \delta_{1}$} \\
\hline$\Delta T_{\max }(h \rightarrow \infty)$ & \multicolumn{2}{|c|}{$48.6 \times 10^{7} \tan \delta_{1}$} & \multicolumn{2}{|c|}{$8.49 \times 10^{7} \tan \delta_{1}$} \\
\hline
\end{tabular}

dielectric interface boundary condition $Q_{\mathrm{SS}}\left(x=D_{\text {int }}\right)=$ 0 , showing that the heat flow indeed depends only on the dissipated power profile. This is expected since, in the steady-state solution, no energy is accumulated in time at any point in space, implying that all the dissipated power must leave the system.

Assuming that the values of the loss tangent are as given in Table II $\left(\tan \delta \sim 10^{-11}\right)$, and even for the repetition rate examined of $1 \mathrm{GHz}$, the temperature rise in the silicazirconia structure is less than $1 \mathrm{~K}$, and the heat flow across the external surface is a fraction of $1 \mathrm{~W} / \mathrm{cm}^{2}$. For comparison, the acceptable technological limiting value of passive heat extraction is of the order of $1500 \mathrm{~W} / \mathrm{cm}^{2}$. Therefore, in the framework of this operation regime, the

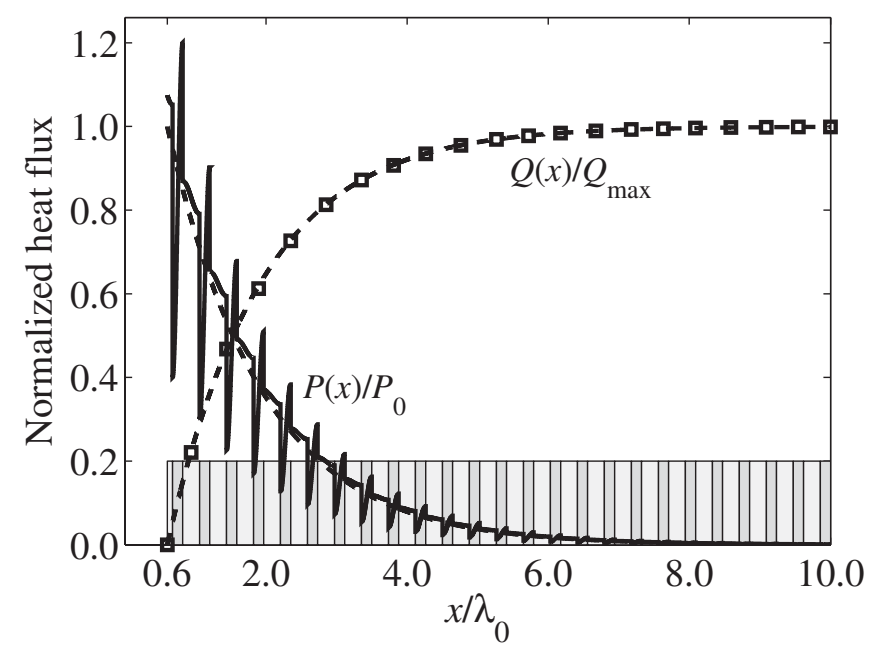

FIG. 6. Heat-flux profile in the silica-zirconia structure, the parameters of which are given in Table II. The dashed line is the approximate curve, and the exact solution is indicated by square markers. The dissipated power profiles, approximate (dashed line) and exact (solid line), are also plotted. The two quantities are normalized by their maximal approximate value. system works orders of magnitude below the limit of heat extraction and well within the range of feasible temperature stabilization. Clearly, if the materials exhibit higher losses by a few orders of magnitude, the temperature rise and the heat flow would attain unacceptable values according to the linear relation presented in the last four rows of Table II.

Silica-silicon. - A structure having a much better confinement and, consequently, higher interaction impedance (see Ref. [5]), is a silica-silicon acceleration structure, having a transverse decay parameter of $x_{c}=0.55 \mu \mathrm{m}$, rather than $x_{c}=2.68 \mu \mathrm{m}$ of the silica-zirconia structure. It follows that, for the silica-zirconia structure, more layers

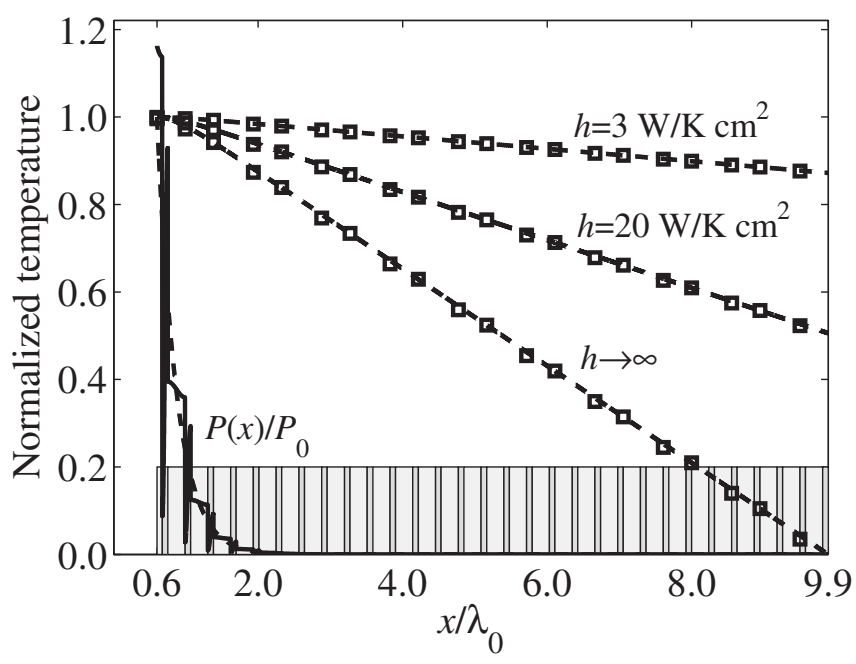

FIG. 7. Temperature profile of different values of $h$ in the silica-silicon structure, the parameters of which are given in Table II. For each value of $h$, the dashed line is the approximate curve, and the exact solution is indicated by square markers. The dissipated power profiles, approximate (dashed line) and exact (solid line), are also plotted. All quantities are normalized by their maximal approximate value. 


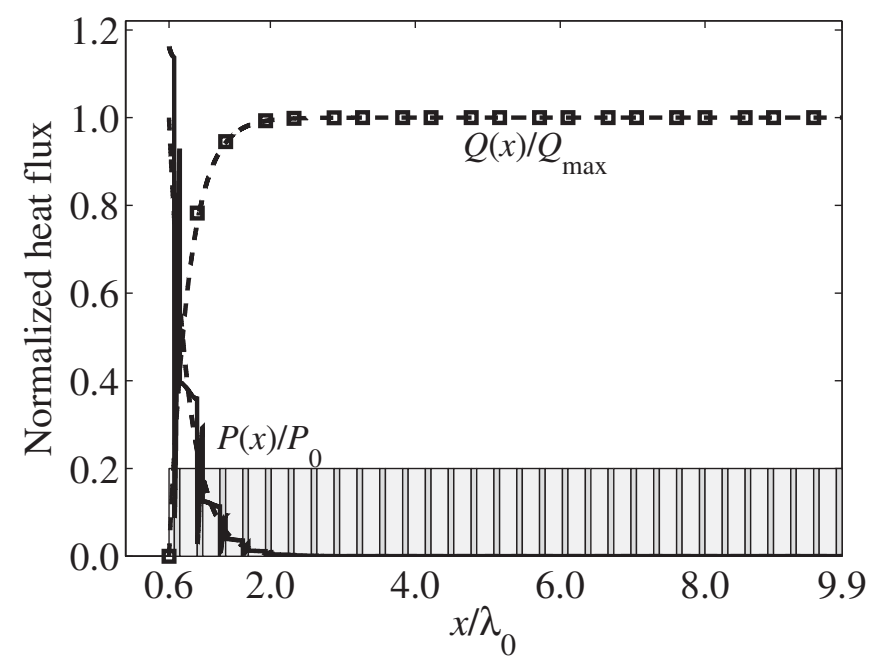

FIG. 8. Heat-flux profile in the silica-silicon structure, the parameters of which are given in Table II. The dashed line is the approximate curve, and the exact solution is indicated by square markers. The dissipated power profiles, approximate (dashed line) and exact (solid line), are also plotted. The two quantities are normalized by their maximal approximate value.

are necessary for a similar confinement to that of the silicasilicon structure. The temperature profile for this structure is given in Fig. 7, and the heat-flux profile is depicted in Fig. 8, while the simulation parameters are presented in Table II. Since the transverse decay of the electromagnetic power is much stronger than that of the silica-zirconia structure, the approximate exponential decay describes less accurately the power fluctuations, and consequently, the factor $\kappa$ [see Eq. (14)] is smaller. As a result, the accuracy of the approximate solutions is also lower; the accuracy of the analytic estimate of the temperature is better than $1 \%$ relative to the temperature maximal value, while the accuracy of the approximate heat-flux solution is better than $10 \%$ relative to its maximal value.

Having assumed throughout the simulations that the acceleration longitudinal field remains constant, it follows that in the silica-silicon structure less power is dissipated, and, consequently, the temperature and the heat flow reach lower values, as can be seen in Table II. This clear preference is further supported by the high thermal conductivity of the silicon.

Two comments are in place before concluding this section. First, it is important to emphasize that no attempt has been made here to optimize materials but rather to demonstrate the main physical scaling laws. For example, a loss parameter of the order of $\tan \delta \sim 10^{-11}$ has been reported for silica. Yet, it is not obvious that in the process of making the Bragg structure this parameter is not altered. However, this should be the focus of an experimental study which is beyond the scope of the present one. Second, it was tacitly assumed that the thermal properties of the bulk material are valid even at an infinitesimal distance from the interface with another material. Deviation from the bulk values of the thermal properties may occur due to misalignments of the two lattices and due to the fabrication process of the dielectric layers. Since, if it occurs, this process takes place in an infinitesimally small volume, we may assume that its relative weight is negligible. A change in the loss tangent at the interfaces may be effectively taken into account by redefining the form factor in Eq. (14).

\section{THERMALLY INDUCED STRESS}

When the temperature distribution across the structure is inhomogeneous, the structure becomes subject to thermal stress. A theoretical foundation of the stress profile in single-layered laser rod and slab geometries has been extensively studied $[11,14,18,24]$. It has been established [11] that, for the temperature distribution of a crosssectional dependence, $\Delta T=\Delta T(x)$, the stress profile is given by

$$
\sigma_{x x}=0, \quad \sigma_{y y}=\sigma_{z z}=\frac{E \alpha}{1-v}\left[\Delta T(x)-\Delta T_{\mathrm{SA}}\right],
$$

where, $E[\mathrm{~Pa}]$ is the elastic modulus, $\alpha\left[\mathrm{K}^{-1}\right]$ is the thermal expansion coefficient, $v$ is Poisson's ratio, and $\Delta T_{\mathrm{SA}}=$ $\frac{1}{D_{\text {ext }}-D_{\text {int }}} \int_{D_{\text {int }}}^{D_{\text {ext }}} \mathrm{d} x \Delta T(x)$ represents the space-averaged temperature. The maximal temperature rise above average which can be tolerated by the material without fracturing is known as the thermal shock resistance and is given by

$$
\delta T_{\max }[\mathrm{K}]=\frac{\sigma_{f}(1-v)}{E \alpha}
$$

with $\sigma_{f}[\mathrm{~Pa}]$ representing the flexure strength, i.e., the maximum stress tolerable by the material. The thermomechanical parameters of fused silica and silicon [50] are listed in Table III.

It is evident from the values listed in Table III that the thermal stress does not pose a severe limitation factor on the system performance with this set of materials. For example, the maximum temperature increase allowed based on thermal stress considerations is more than 3 orders of magnitude larger than the anticipated temperature increase.

When dealing with multilayered structures, a possible stress induced limitation factor may occur due to the mis-

TABLE III. Thermomechanical properties of fused silica and silicon [50].

\begin{tabular}{lcc}
\hline \hline & Fused $\mathrm{SiO}_{2}$ & $\mathrm{Si}$ \\
\hline$E[\mathrm{GPa}]$ & 72.6 & 162 \\
$\alpha\left[10^{-6} \mathrm{~K}^{-1}\right]$ & 0.51 & 2.62 \\
$v$ & 0.164 & 0.22 \\
$\sigma_{f}[\mathrm{MPa}]$ & 110 & 130 \\
$\delta T_{\max }[\mathrm{K}]$ & 2483.7 & 238.9 \\
\hline \hline
\end{tabular}


match of the thermomechanical properties on both sides of the interface between the inner layers, which may create cracks. Relaxation of the thermal mismatch induced stress [51] and designing crack-free Bragg mirrors [52,53] were addressed in recent years. In the case of a Bragg acceleration structure, the temperature where these effects become significant is prohibitively high from the perspective of radiation confinement and phase control, due to changes in the dielectric coefficients, since the change of the refractive index with the temperature, $\partial n / \partial T$, is of the order of $10^{-4}-10^{-5} \mathrm{~K}^{-1}[54,55]$.

\section{CONCLUSION}

In conclusion, we have analyzed the temperature and heat flow across the planar optical Bragg acceleration structure. An approximate dynamic analysis showed that, for the range of parameters of interest, it is sufficient to consider the steady-state problem, rather than account for high-repetition rate pulses. Approximate analytic expressions for the steady-state temperature and heat variations were given [Eq. (12)]. These expressions were tested against the exact solutions and their accuracy was found to be better than $1 \%$ for the temperature, and better than $10 \%$ for the heat flux, relative to their respective maximal values. With these approximate solutions, it is possible to estimate, accounting only effectively for the dielectric layers, the maximal change in temperature at the internal boundary and the heat flow at the external boundary. Assuming an accelerating gradient of $1 \mathrm{GV} / \mathrm{m}$ and a low-loss material similar to that existing in communication optical fibers $\alpha \sim 1 \mathrm{~dB} / \mathrm{km}$, resulting in a loss tangent of the order of $\tan \delta \sim 10^{-11}$, the temperature increase is less than $1 \mathrm{~K}$, and the heat flow is of the order of $1 \mathrm{~W} / \mathrm{cm}^{2}$. This heat flow is 3 orders of magnitude lower than the known technological limit, implying that a proper choice of materials may eliminate thermal considerations from the list of obstacles for the operation of an optical accelerator. In this regard, the wavelength, which we have taken arbitrarily to be $1 \mu \mathrm{m}$, is another important parameter that should be optimized in order to achieve the lowest dissipation possible in the materials involved.

\section{ACKNOWLEDGMENTS}

This study was supported by the United States Department of Energy.

[1] Y.C. Huang, D. Zheng, W. M. Tulloch, and R. L. Byer, Appl. Phys. Lett. 68, 753 (1996).

[2] J. D. Lawson, IEEE Trans. Nucl. Sci. 26, 4217 (1979).

[3] P. M. Woodward, J. IEE 93, 1554 (1947).

[4] X. E. Lin, Phys. Rev. ST Accel. Beams 4, 051301 (2001).

[5] A. Mizrahi and L. Schächter, Phys. Rev. E 70, 016505 (2004).
[6] R. P. Larsen and A. A. Oliner, in IEEE-MTT International Microwave Symposium Digest (IEEE, Boston, MA, 1967), Vol. 67, pp. 17-22.

[7] P. Yeh and A. Yariv, Opt. Commun. 19, 427 (1976).

[8] P. Yeh, A. Yariv, and E. Marom, J. Opt. Soc. Am. 68, 1196 (1978).

[9] S. Banna, D. Schieber, and L. Schächter, Appl. Phys. Lett. 84, 723 (2004).

[10] S. Banna, D. Schieber, and L. Schächter, J. Appl. Phys. 95, 4415 (2004).

[11] J. M. Eggleston, T. J. Kane, K. Kuhn, J. Unternahrer, and R. L. Byer, IEEE J. Quantum Electron. 20, 289 (1984).

[12] T. J. Kane, J.M. Eggleston, and R. L. Byer, IEEE J. Quantum Electron. 21, 1195 (1985).

[13] R. Weber, B. Neuenschwander, and H. P. Weber, Opt. Mater. 11, 245 (1999).

[14] D.C. Brown and H.J. Hoffman, IEEE J. Quantum Electron. 37, 207 (2001).

[15] M. Schmid, R. Weber, T. Graf, M. Roos, and H. P. Weber, IEEE J. Quantum Electron. 36, 620 (2000).

[16] M. Schmid, T. Graf, and H. P. Weber, J. Opt. Soc. Am. B 17, 1398 (2000).

[17] A. McInnes and J. Richards, IEEE J. Quantum Electron. 32, 1243 (1996).

[18] R. Paschotta, J. Aus der Au, and U. Keller, IEEE J. Sel. Top. Quantum Electron. 6, 636 (2000).

[19] R. Weber, B. Neuenschwander, M. Mac Donald, M. B. Roos, and H. P. Weber, IEEE J. Quantum Electron. 34, 1046 (1998).

[20] Y. Tzuk, A. Tal, S. Goldring, Y. Glick, E. Lebiush, G. Kaufman, and R. Lavi, IEEE J. Quantum Electron. 40, 262 (2004).

[21] J.E. Graebner, J.A. Mucha, L. Seibles, and G. W. Kammlott, J. Appl. Phys. 71, 3143 (1992).

[22] J.E. Graebner, S. Jin, G. W. Kammlott, J. A. Herb, and C. F. Gardinier, Appl. Phys. Lett. 60, 1576 (1992).

[23] W. Xie, S. C. Tam, H. Yang, J. Gu, G. Zhao, Y. L. Lam, and C.H. Kam, Opt. Laser Technol. 31, 387 (1999).

[24] W. Xie, S. C. Tam, H. Yang, J. Gu, G. Zhao, Y. L. Lam, and W. Tan, Opt. Laser Technol. 31, 521 (1999).

[25] W. Xie, S. C. Tam, Y. L. Lam, H. Yang, J. Liu, J. Gu, and W. Tan, Opt. Laser Technol. 32, 193 (2000).

[26] W. Koechner, J. Appl. Phys. 44, 3162 (1973).

[27] U. O. Farrukh, A. M. Buoncristiani, and C. E. Byvik, IEEE J. Quantum Electron. 24, 2253 (1988).

[28] U. O. Farrukh and P. Brockman, Appl. Opt. 32, 2075 (1993).

[29] P. Hello, E. Durand, P. K. Fritschel, and C. N. Man, J. Mod. Opt. 41, 1371 (1994).

[30] B. A. Usievich, V.A. Sychugov, F. Pigeon, and A. Tishchenko, IEEE J. Quantum Electron. 37, 1210 (2001).

[31] M.L. Burgener and R.E. Reedy, J. Appl. Phys. 53, 4357 (1982).

[32] R. J. Anderson, J. Appl. Phys. 64, 6639 (1988).

[33] M. R. Madison and T. W. McDaniel, J. Appl. Phys. 66, 5738 (1989).

[34] O. W. Shih, J. Appl. Phys. 75, 4382 (1994).

[35] W. A. McGahan and K. D. Cole, J. Appl. Phys. 72, 1362 (1992).

[36] N.D. Milošević and M. Raynaud, Int. J. Heat Mass Transfer 47, 1627 (2004). 
[37] A. Haji-Sheikh and J. V. Beck, Int. J. Heat Mass Transfer 45, 1865 (2002).

[38] A. Haji-Sheikh, J. V. Beck, and D. Agonafer, Int. J. Heat Mass Transfer 46, 2363 (2003).

[39] D. H. Y. Yen and J. V. Beck, J. Eng. Math. 49, 305 (2004).

[40] F. de Monte, Int. J. Heat Mass Transfer 45, 1333 (2002).

[41] J. R. Ho, C. P. Kuo, and W. S. Jiaung, Int. J. Heat Mass Transfer 46, 55 (2003).

[42] J. R. Miller and P. M. Weaver, Composite Structures 59, 267 (2003).

[43] Y. Sun and I. S. Wichman, Int. J. Heat Mass Transfer 47, 1555 (2004).

[44] E. N. Wang, L. Zhang, L. Jiang, J. M. Koo, J. G. Maveety, E. A. Sanchez, K.E. Goodson, and T.W. Kenny, J. Microelectromech. Syst. 13, 833 (2004).

[45] S. G. Kandlikar and W. J. Grande, Heat transfer engineering 24, 3 (2003).

[46] R. L. Webb, S. Yamauchi, S. Denko, and K. K. Tochigi, IEEE Trans. Components Packag. Technol. 25, 608 (2002).
[47] A. G. Fedorov and R. Viskanta, Int. J. Heat Mass Transfer 43, 399 (2000).

[48] A. Yariv, Optical Electronics in Modern Communications (Oxford University Press, New York, 1997), 5th ed.

[49] K. Nagayama, M. Kakui, M. Matsui, T. Saitoh, and Y. Chigusa, Electron. Lett. 38, 1168 (2002).

[50] M. Bass, E. W. Van Stryland, D. R. Williams, and W. L. Wolfe, Handbook of Optics, Devices, Measurements and Properties (McGraw-Hill, New York, 1995), Vol. II.

[51] H. Zogg, S. Blunier, A. Fach, C. Maissen, P. Müller, S. Teodoropol, V. Meyer, G. Kostorz, A. Dommann, and T. Richmond, Phys. Rev. B 50, 10801 (1994).

[52] Z. Shi, H. Zogg, P. Müller, I. D. Jung, and U. Keller, Appl. Phys. Lett. 69, 3474 (1996).

[53] S. Schön, H. Zogg, and U. Keller, J. Cryst. Growth 201202, 1020 (1999).

[54] G. Ghosh, IEEE Photonics Technol. Lett. 6, 431 (1994).

[55] F. G. Della Corte, M. Esposito Montefusco, L. Moretti, I. Rendina, and G. Cocorullo, J. Appl. Phys. 88, 7115 (2000). 